DOI: 10.1515/ausp-2015-0027

\title{
Die rumänischen Identitäten in Veränderung
}

\author{
Béla BÍRÓ \\ Sapientia Universität, Fakultät für Geisteswissenschaften \\ birobela@sapientia.siculorum.ro
}

\begin{abstract}
Abstrakt. Der vorliegende Beitrag setzt sich mit dem in den Sozialwissenschaften recht umstrittenen Konzept der Identität auseinander. Dabei wird versucht, durch eine Analyse die sich auf ein flüssiges, zeit- und geschichtsimmanentes Verständnis von Identität baut, auf eine Morphologie von Identitätskonstruktionen zu stoßen. Als Beispiel und Objekt der vorliegenden Analyse dienen die Beziehung und das Zusammenleben der Minderheiten in Rumänien, vor allem während des kommunistischen Regimes.
\end{abstract}

Schlüsselwörter: Identität, Alterität, Minderheit, Kommunismus, Rumänien

Abstract. The following paper looks into a heavily disputed concept within
the social sciences, that of identity. The goal of the paper is to identify a
morphology of identity, as a social phenomenon, by employing an analysis
based on a fluid and historicist understanding of identity. The relationship
and cohabitation of the ethnic groups in Romania, especially during the
communist regime, serves both as example and study object of the analysis.

Keywords: identity, difference, minority, communism, Romania

Identität ist eine der strittigsten Begriffe der Sozialwissenschaften. Es gibt eine große Menge von essentialistischen und nominalistischen Definitionen, die von Anfang des theoretischen Denkens rivalisieren. Der ersten Klasse gemäß sind Identitäten zeitlose Grundeigenschaften des menschlichen Geistes, der zweiten gemäß sind sie verschiedene und mehr oder minder angemessene Antworten an die geschichtlichen Herausforderungen des sich stetig verändernden gesellschaftlichen Seins, und als solche entwickeln sie sich in der Zeit.

Die neueste Literatur verwendet den Begriff fast ausschließlich in dem zweiten Sinn (Dubar 2000; Kymlicka-Straehle 1999: 65-88; Connor 1972: 319-355; Held 1995) Identität erscheint als ein wechselseitiges Spiel ständiger Differenzierung und Identifizierung. Die erste grenzt die Individualität von anderen Individualitäten (d. h. Alterititäten) ab. Die zweite stellt die gemeinsamen physischen, psychischen und geistlichen Eigenschaften der Individuen einer Kollektivität fest. Identität ist also eine sich verändernde gemeinsame Zugehörigkeit. Daher das 
gut bekannte Paradoxon der Identität: das Unteilbare ist das Teilbare. Das Paradoxon ist nur im Fall lösbar, wenn wir das gemeinsame Element der beiden Operationen, die Identifikation der Anderen und die Selbstidentifikation durch den Anderen in Betracht nehmen. Es gibt keine Identität ohne Non-Identität, anders gesagt ohne Verschiedenheit. Und umgekehrt. Das ist auch im geschichtlichen Bezug wahr. Genau wie Alteritäten, ändern sich Identitäten - unter verschiedenen geschichtlichen Kontexten und Bedingungen - auch.

Es gibt also zwei grundlegende Typen von Identifikationen: Identitäten für sich (oder Identifikationen, die uns von anderen spontanerweise beigelegt werden) und Identitäten für andere (oder Identifikationen, die wir von den anderen für uns - oft mittels verschiedener Formen des gesellschaftlichen Zwanges - beanspruchen). Diese zwei Grundtypen der Identifikation beeinflussen sich wechselseitig. In jeder Gemeinschaft, Gesellschaft, in jedem Verein usw. gibt es eine permanente, aber meist latente Debatte über diese Identitätsfragen. Die Individuen und die Kollektivität lassen sich immer auf scharfe Unterhandlungen ein, bis die Identifikation für sich die Identifikation für das Andere genau überdeckt. Wenn diese Überdekkung - aus verschiedenen Ursachen - unmöglich ist, entstehen die sogenannte Anomalien oder Krisen des Identitätsbewusstseins, die gewohnheitsmäßig durch kognitiven Dissonanzen (in der Gesellschaft allgemein akzeptierte und als Wahrheit betrachtete Fantasieprodukte) aufgehoben werden.

Eine solche Polemik hat von Anfang an rumänische Identitäten markiert. Die Rumänen sind von ihren Nachbarn und nicht-rumänischen Mitbürgern (in erster Reihe von Ungarn und Deutschen) als Einwanderer betrachtet, aber die Rumänen haben im 19. Jahrhundert ein ganz anderes Selbstbewusstsein entwickelt, sie betrachten sich auch heute als die alteingesessene Urbevölkerung des Landgebietes von Rumänien.

Die Problematik hatte keine sonderbare Bedeutung, bis die ungarische und bald die rumänische politische Elite diese Identifikationen zu politischen Grundlegitimationsformen zu entwickeln begann und zu den politischen Prinzipien des Staatverwaltens deklarierte. Die zwei verschiedenen Identifikationsvarianten waren kategorisch unvereinbar, weil sie ausschließlich die einseitigen Machtansprüche begründen mussten, die auf die gestrittenen Territorien (Siebenbürgen, Bessarabien, Nordbukowina, Süddobrogea usw.) erhoben worden waren. Dieser Identifikationsabgrund hat sowohl die Rumänen, wie auch die Ungarn, Deutschen und die anderen Bevölkerungen zur Entwicklung gegenseitiger mythischer Narrativen gezwungen. Aber darüber sprechen wir später.

Die Hauptidee dieses Beitrags ist also der Satz, dass die Identifikationsformen der Kollektivität wie auch der Individuen selbst in der Zeit sich stetig verändern. Claude Dubar fixiert in seinem Buch Die Krise der Identitäten - auf Grund einer Typisierung entwickelt von Max Weber - zwei geschichtliche Grundformen der Identifizierung: die gemeinschaftliche (communitaire) und die gesellschaftliche (societaire) Identifikation. 
Die Vertreter der ersten Variante setzten den Glauben an der Existenz einiger Gruppen, „Gemeinschaften“ (communauté) genannt, voraus, die als einige Systeme von Plätzen und von - der Individuen vorzuschreibenden und von Generation zu Generation unverändert weitergegebenen - Namen betrachtet sind.

Die Vertreter der zweiten begründen ihre Definitionen durch die Existenz einiger vielfachen, sich verändernden, vergänglichen Kommunität, zu der die Individuen sich nur für begrenzte Dauer beigesellen und die den Mitgliedern eine Reihe von Identifikationsquellen liefert, die von ihnen verschiedenerweise und provisorisch gebraucht wird.

Diese zwei Grundtypen schließen sich nicht aus, vielmehr ergänzen sie sich. Die Verwandlungen der Identität treten aber auf Grund eines stabilen kulturellen Unterbaus ein. Auch dieser Unterbau ist verwechselbar, aber der Versuch kann nur selten unmittelbar „erfolgreich“ bezeigen. In der ersten Generation erscheint das Versuch regelrecht erfolglos, vielmal eben traumatisierend. Man braucht solide und möglichst im Kindesalter zu eigen gemachte Kultur- und Sprachkenntnisse, um diesen Identitätswechsel mit Erfolg zu durchqueren. Natürlich gibt es auch wichtige Ausnahmen. Aber nur sehr selten.

Im Allgemeinen sind diese Grundkategorien - als Max Webersche Idealtypen betrachtet - gut verwendbar. Ihre Anwesenheit ist auch in der Entwicklung des rumänischen Identitätsbewusstseins nachweisbar.

Die Rumänen lebten vom Spätmittelalter bis Ende der Neuzeit jahrhundertelang in drei verschiedenen Reichen: in der Habsburgischen Monarchie (Siebenbürgen, Banat und Partium), im Russischen Reich (Moldavien, Bessarabien, Bukowina) und im türkischen Sultanat (Oltenien, Muntenien und Dobrogea). Auf Grund der - von der vorausgesetzten gemeinsamen Abstammung und Sprachgemeinschaft definierte - gemeinsamen Bewusstseinszüge, entwickelten sich auf diesen - voneinander politisch getrennten - Territorien, in den sogenannten rumänischen Prinzipaten auch sehr verschiedene regionale Sondereigenschaften. Die rumänische Fachliteratur spricht von einer sonderbaren Akkommodationsfähigkeit der rumänischen Bevölkerung, aber auch von einer sonderbaren Fähigkeit zum Aufheben des meist oberflächlichen Akkommodationsmusters nach dem Ende der verschiedenen Fremdherrschaften. Das Bewusstsein des römischen Ursprungs, der lateinischen Herkunft der rumänischen Sprache und der orthodoxen Zugehörigkeit ${ }^{1}$ aller Rumänen erwies sich als eine außergewöhnlich mächtige Kohäsionskraft, ein festes gemeinschaftliches Bund. (Die griechischkatholische Kirche Siebenbürgens ist ein sehr spätes und nur unter Habsburgischem Zwang eingetretenes Ereignis.)

C. Rădulescu-Motru, einer der hellsichtigsten Köpfe der ersten Hälfte des 20. Jahrhunderts stellt in seinem berühmten Buch Etnicul românesc (Die rumänische Ethnizität) drei aufeinanderfolgende Entwicklungsformen des ethnischen

1 Dazu siehe das berühmte Buch von Man, Gloria: The Ortodox Fault Line. 
Gemeinschaftsbewusstseins fest: die Stammungsgemeinschaft, die Sprachgemeinschaft und die Schicksalsgemeinschaft. Die letzteren Formen, meint er, enthalten immer auch die vorstehenden. Dieser Logik gemäß ist die Schicksalsgemeinschaft die höchste Form der etnischen Identität, die Rădulescu-Motru als gleichberechtigtes Synonym für die nationale Identität betrachtet. Sein Werk ist während des Zweiten Weltkriegs entstanden, und trägt an sich den starken Einfluss der damaligen deutschen Geopolitik.

Diese Betrachtungsweise beeinflusst auch im Folgenden die einflussreichsten Vertreter des rumänischen Nationalismus. Die Idee der großzügigen nationalen Berufung ist eine Konstante des rumänischen nationalen Denkens geworden.

Der Dichter und Philosoph Lucian Blaga hat daraus eine umgreifende nationale Metaphysik erarbeitet, die Theorie des sogenannten mioritischen Raumes. Blaga, der ein guter Kenner der deutschen Sprache und der zeitgenössischen deutschen Nationalphilosophie und Anthropologie, der Werken von Alois Riegl, Frobenius und anderen war, stellte zwischen den visuellen Eigenschaften der siebenbürgischen Landschaft (die in der Sicht der rumänischen Nationalisten die Wiege des rumänischen Volkes sein musste) und den seelischen Zügen, Betrachtung- und Wahrnehmungsweise des rumänischen Bauers einen Zusammenhang fest. Das charakteristische Sinneswahrnehmungsmuster des Rumänen, meinte Blaga, ist die Ondulation, die Wellenlinie, die die Berg-auf-Tal-wärts-Bewegung der siebenbürgischen Hochebene modelliert. Diese Konzeption gründete auf die berühmteste rumänische Volksballade Mioritza, in der die zwei Hirten aus Ungarn und aus Rumänien ihren moldawischen Kollegen, um seine Güter zu erwerben, ermordet haben. Die Ballade selbst enthält nichts Nationalistisches, die zwei Mörder sind Rumänen als auch der Ermordete selbst, der den Tod ohne keinerlei Verteidigungsversuch fatalistisch akzeptiert und erwartet, obwohl er von seinem Lieblingslämmchen über die lauernde Gefahr rechtzeitig gewarnt wurde.

Der Tatbestand der Ballade wurde von dem nationalistischen Metaphysiker in enger Zusammenarbeit mit seinem französischen Kollegen vielseitig ausgebeutet (Miskolczy 1994). Das Symbol des unschuldigen rumänischen Opfers ist ein mächtiges Element zum Hochspannen der Sympathie für die rumänische Nationalangelegenheit.

Dieses schöne, metaphysisch-poetische Bild des milden, fatalistischen, im unendlichen und ewigen Kosmos (in der sogenannten kosmischen Ondulation) eingebetteten Bauern war für die Realisierung der Nationaleinheit sehr gut geeignet, aber für eine weitere hochmütige nationale Berufung, für die Verwirklichung des einheitlichen, homogenen, einsprachigen Nationalstaates erscheint es weitgehend ungeeignet. Die rumänische Glorie benötigt viel tätigere Nationaleigenschaften.

Die Kriegsgefahr und die Versuche zur Zwangsassimilation förderten die schnelle Erarbeitung eines effizienten Heldenmythos und die Wiedervereinigung der rumänischen Nationalkirche. Die griechisch-katholische Konfession wurde 
verboten. Die Armee und die Kirche wurden auch unter der kommunistischen Herrschaft als die wichtigsten Nationalinstituten verehrt und geschätzt. Nach dem Zweiten Weltkrieg wurde die Kirche teilweise von der kommunistischen Partei ersetzt. Die Armee und der Heldenmythos aber bleibt. Die Porträtgalerie der Nationalhelden endete natürlicherweise mit dem Bild des großen Führers, N. Ceauşescu. Dieser Vorgang wurde im Lucian Boias berühmten Buch: Istorie şi mit în conştiința românească (Geschichte und Mythos im rumänischen Selbstbewusstsein) wunderbar geschildert. Boia argumentiert am rumänischen Material die berühmte Thesis von Benedict Anderson, dass Nationen - mindestens in großem Masse - „eingebildete Kollektivitäten“ (Anderson 1991) seien.

Die Kraftanstrengungen zum Aufbau des Nationalstaates haben ihre Absurdität unter Ceauşescus nationalkommunistischer Diktatur erreicht. Die übermäßige Lobhudelei lenkte die Aufmerksamkeit der verantwortlichen Intellektuellen immer mehr an die nackte Realität, auf das Ausgeliefertsein, die Obedienz und Demütigung der rumänischen Gesellschaft. Die Worte - in der Öffentlichkeit ausgesprochen - wechselten ihre Bedeutung fast systematisch: das Heldentum begann Feigheit, die Freiheit Eroberung, der nationale Stolz nationale Scham zu bedeuten. Die selbstkritische Ironie des oppositionell gesellten rumänischen Intellektuellen, der immer sehr entwickelt war, begann noch einmal aufzublühen.

Die rumänische Seele ist immer durch einen scharfen Dualismus gekennzeichnet. Eminescu und Caragiale, die größten Genies des 19. Jahrhunderts haben eine unbarmherzige Kritik über die rumänischen Zustände ausgeübt. Aber ihre Kritik wurde auf ganz verschiedene Betrachtungsweisen begründet. Eminescu - der größte Nationaldichter - vertrat eine krasse Xenophobie und Antisemitismus. Caragiale - der größte Dramatiker und Kosmopolit - hat in seinen genialen Komödien und Kurzgeschichten eben diesen rumänischen Nationalismus, die Plauderei, die Heldenpose verhöhnt.

Nach dem Fall Ceauseşcus wählte sich die einflussreichste satirische Wochenzeitung Rumäniens als Titel den Namen der berühmtesten Figur dieser Komödien, Cațavencu, der sich als Liberal deklarierte, aber seine politischen Ansichten ganz ohne politische Überzeugung seinen persönlichen Interessen entsprechend ständig wechselt. Er hat einen einzigen echten Charakterzug, den hemmungslosen Willen zur Macht. Am Ende wird er besiegt, aber die Sieger sind noch niederträchtiger als er. In dieser Welt der Lüge, Gemeinheit, Verderbtheit gibt es einen einzigen ehrlichen Mann, den sogenannten „,besoffenen Staatsbürger“ („,cetățean turmentat"), denn in dieser Welt, wenn du ehrlich bleiben willst, gibt Caragiale $\mathrm{zu}$, musst du stätig betrunken sein.

Ein anderes Meisterwerk, das die Unverschämtheit, die schmutzige moralische Gemeinheit dieser Welt entlarvt, ist von einem anderen Caragiale, von seinem Sohn Mateiu geschrieben. Der Roman Craii de curtea veche, dessen Titel kaum übersetzbar ist (etwa: Die Schurken aus dem alten Hof/Die Ritter vom Alten 
Hoff, hat auch eine positive Figur, eine fast immer betrunkene Frau, die ihren Bräutigam, einen russischen Herzog, im Unabhängigkeitskrieg von 1877 verloren hat. Sie, die ewig Betrunkene ist die reinste Figur des Romans, die rumänische Frau, eine aus der Menge, in die sich auch ein hoch raffinierter russischer Herzog tödlich verlieben kann.

Nach 1989 ist die berühmte Frage von Cioran: „Wie kann jemand Rumäne sein?" nochmals aktuell geworden. Ciorans Frage ist im Grunde genommen die umgekehrte Variante der berühmten Frage von Montesquieu: „Wie kann man Perser sein?" Horia-Radu Patapievici, einer der besten Publizisten der neuen Generation, fixiert in einem scharfsinnigen Essay, mit dem Titel Despre faptul de a fi român (Über die Tatsache Rumäne zu sein) die drei impliziten Voraussetzungen, die zum Formulierung der Frage führen konnten. 1. Die implizite Voraussetzung der Parisianer, für die, Franzose zu sein, der natürlichste aller Dinge sei. 2. Die implizite Voraussetzung des Verfassers (Montesquieu), für den die Selbstverständlichkeit des Französisch-seins nicht mehr unproblematisch ist. Und 3. die implizite Voraussetzung des kulturellen Kontextes, in dem die zwei vorigen Fragen schon formulierbar sind. Aufgrund der Analyse von diesen Voraussetzungen konkludiert Patapievici: „In den Verständlichkeitsvoraussetzungen der an den Persern gerichteten Frage sind alle Möglichkeitsbedingungen zum Aufbau der modernen Identität anwesend.“ (Patapievici 2004: 45)

Im Folgenden examiniert er die implizite Voraussetzungen der cioranschen Frage. Der Grundgegensatz der im Hintergrund der Fragestellung steht, ist Vitalität vs. Dekadenz. Es gibt zwei Grundtypen der Identität: starke und schwache. Die starken Identitäten haben zur Folge Erfolgsverhalten, die schwachen aber Misserfolgsverhalten. Die Gemeinschaften, deren Individuen von den schwachen Identitäten beeinflusst sind, erleiden nur die Geschichte, deren Helden immer die Stärkeren sind.

1990 meint Cioran auch, dass „Rumäne zu sein, ist ein Bann...“ Aber er erklärt schon 1936 (noch in Rumänien): „Der Existenzmodus des Rumänen ist die Kleinigkeit...“ und „Der Diminutiv ist unsere Divinität“. Die Rumänen, sagt er, sind so mittelmäßig, dass sie mit ihrer Mittelmäßigkeit auch die Juden, die zwischen ihnen lebten, kontaminiert haben. Bei uns gibt es kein Kaffka oder Canetti. Rumänien, meint er, ist ein second hand Land, das letzte Land der Welt. (Patapievici 2004: 47)

Cioran brandmarkt die rumänische Identität, weil sie diese mit den anderen, von ihm hochgeschätzten Identitäten gegeneinander stellt. Er lässt außer Betrachtung, dass von innen gesehen auch diese starke Identitäten sehr niedrig erscheinen könnten. Sieh die Meinung von Nietzsche über die Deutschen, die von Dostojewski über die Russen, die von Solschenizyn über die Sowjetleute usw. „Ich wollte - schrieb Cioran -, dass [die rumänische Identität - B. B.] stark, unmäßig, wahnsinnig, gleich einer bösartigen Kraft, einer Fatalität sei, die die Welt zittern 
lässt, sie war aber klein, unerheblich, ohne die Eigenschaften, die ein großartiges Schicksal darstellen könnten." (Patapievici 2004: 49)

Er leidet wegen der angeblichen Nichtsnutzigkeit, dem Unernst, der Geringfügigkeit - anders gesagt wegen den von Caragiale geschilderten Eigenschaften der Rumänen. Die rumänische Frage bedeutet für Cioran die Notwendigkeit der Assimilierung einer starken (d. h. westlichen) Identität, durch die die Rumänen von dem Minorat zum Majorat hingelangen, anders gesagt, endlich einmal emporwachsen könnten. Das ist nur mittels einer Zwangsmodernisierung möglich. Also Ciorans Frage gibt die Möglichkeitsbedingungen der Modernisierung der premodernen oder noch nicht genug modernisierten Identitäten.

Diese Fragestellung lässt uns verstehen, wie die kommunistische Diktatur in Rumänien so hässliche Erscheinungsformen ausgestalten könnte. Diese Vergötterung der Kraft war ein sehr geeigneter Boden für allerlei Gewaltherrschaft. Eine peripherische Identität lässt sich nämlich in eine zentrale nur durch Blut und Asche, Kraft und Gewalt verändern.

Patapievici glaubt nicht mehr, dass allein Rumänien heute noch imstande wäre, eine sogenannte starke Identität zu realisieren. Wir müssen uns in die EU integrieren, aber das bedeutet nicht, dass wir dem Traum der starken Identität absagen müssen, wir müssen eher auf der Hut sein. „Man sagt uns heute, dass die wirklich demokratische, die wirklich moderne Welt hätte den Begriff der starken Identität abandonniert. Es ist falsch. Der Westen hat die starke Identität überhaupt nicht abandonniert. Er hat nur den Grundsatz der Veränderung und der Annahme der verschiedenen Identitäten ad libitum akzeptiert.“- schreibt er 2004 und fügt noch hinzu: „Wir wollen uns keine kommunitäre, also tief eingewurzelte Identitäten aufbauen, sondern nur individuelle, nach Lust und Laune jedermanns. Man muntert uns auf, um uns persönlich nach Belieben zu verhalten, müssen wir aber in dem öffentlichen Raum die Spielregeln strikt respektieren.“ (Patapievici 2004: 59)

Die Schlussfolgerung: „Die starke Identitäten sind nicht tot, sie haben den Meinungspluralismus, die lange Ephemerität, die kulturelle Diversität und den Voluntarismus akzeptiert. Um ihre Stärke zu bewahren, haben sie sich für schwach erklärt. Sie haben Teil am Schicksal der Zivilisationen: sie wissen, dass sie sterblich sind, aber sie haben die Prätention ganz lebend zu sein, bis sie noch leben. Die schwache Identitäten sind heute eifersüchtiger als je.“

Aber das scheint jedoch eine andersartige „starke Identität“ zu sein. Um solche starke Identitäten zu haben, müssen wir unsere politischen Institutionen und nicht die Grenzen oder die ethnischen Verhältnisse der Gesellschaft ändern. Entsprechende Institutionen sind imstande auch die Grundeigenschaften der Kollektivität zu ändern. Die Rumänen müssen den Glauben an ihrer Vorzüglichkeit abandonnieren.

In dieser Akzeptanz reduziert sich die Identitätsfrage an die Frage nach dem Wesen der Institutionen. Wie sind diese Institutionen, wie sind sie entstanden? 
Um den Antwort zu finden, müssen wir bis die Gründung des heutigen rumänischen Staates, dem sogenannten Großrumänien zurückgehen.

Die rumänische Gesellschaft musste damals zwischen zwei grundlegenden Möglichkeiten, zwei Typen von politischer Legitimität wählen. Es ist allbekannt, dass die Stabilität eines Staates sich nur auf eine von den Staatsbürgern freiwillig akzeptierte Legitimation begründen kann. Falls die Legitimation nur partial oder formal sei, kann das Gleichgewicht der Gesellschaft und folgenderweise des Staates nur durch Gewalt bewahrt werden.

Die Grundvoraussetzung der freiwillig akzeptierten Legitimation ist aber die völlige Gleichheit der Bürger. In einem sogenannten Nationalstaat, der sich in einen Aufbauprozess der einheitlichen Nation (Englisch gesagt Nationbuilding) engagiert, ist das ganz unmöglich, weil dieser Prozess eben die Abschaffung der anderen nationalen Gemeinschaften voraussetzt.

Die Folgen sind oft unübersehbar. Wenn die Legalität im Interesse verschiedener gesellschaftlichen Gruppen - aufgrund sozialer oder geschichtlich-nationaler Erwägungen - rechtmäßig aufgehoben wird, wird sich die Zweideutigkeit in eine unerschöpfliche Quelle aller Betrügereien, des Schwindels, von Missbräuchen, oder vielmehr von „offiziellen“ Rachen und Vergeltungen verwandeln. Wenn man die Güter einiger gesellschaftlichen Gruppen „rechtmäßig“ beschlagnahmen kann, wenn man die Meinungsfreiheit legal aufheben möchte, werden die Güter und die Freiheit aller Staatsbürger, gar die gesellschaftliche Ordnung selbst, in die größte Gefahr geraten. Es wird genügend, jemanden mit anationalem oder antisozialem Verhalten anzuschuldigen, um zum Opfer allerlei Missbräuche zu fallen. Die nationalstaatliche Lizitation erschafft unerschöpfliche Möglichkeiten für die „guten Rumänen“, um die Öffentlichkeit hemmungslos zu erobern, um die „wenig guten Rumänen“ aus ihrer Stellungen oder gar aus ihrer Wohnungen zu verdrängen. Von den „schlechten Rumänen“ nicht zu sprechen!

Die Schöpfer des großen rumänischen Staates mussten zwischen zwei großen Möglichkeiten wählen: zwischen der demokratischen, anders gesagt auf dem nationalen Konsensus begründeten Legitimation und zwischen der autoritären, anders gesagt auf geschichtlichen und nationalen Prinzipien begründeten Legitimation. Im ersten Fall, wenn die Nation eine - die kulturellen Gemeinschaften des Staates integrierende - politische Gesellschaft bedeutet, ist das Ergebnis die echte Gleichheit der Staatsbürger. Im zweiten, wenn die Nation eine - die kulturellen Gemeinschaften des Staates assimilierende - politische Gemeinschaft bedeutet, ist das Ergebnis die Ungleichheit der Staatsbürger.

Die erste Variante hätte in der Lage der in den rumänischen Staat geratenen Minderheiten, die Ungarn, Sachsen, Juden, Serben, Türken, Russen, Bulgaren usw. keine schmerzliche Veränderung verursacht. Die Ungarn aus Siebenbürgen und teilweise auch die Sachsen und Juden hätten ihre vorherige Sonderrechte und Privilegien verloren. Die Rumänen dagegen hätten selbstverständlich die 
Möglichkeit gewonnen, ihre nationale Institutionen, eigene Städte oder Stadtzentren auszubauen, die eine günstige, wohlangebrachte Konkurrenz für die vorherigen ungarischen, deutschen und anderen Institutionen und Städten hätten einführen können. Auf wechselseitige Kompromisse gebaut, konservierte sich die Vielfältigkeit von schweizerischem Typ der siebenbürgischen Gesellschaft, und aufgrund der autonomen und dezentralisierten Ordnung mochten sich auch die rumänischen Gebiete Siebenbürgens und der anderen rumänischen Ländern das sehr ehrliche Niveau der ehemaligen Habsburgischen Monarchie erreichen. Die Rumänen konnten alle ihre Forderungen und Ansprüche verwirklichen, ohne dass die Interessen der Ungarn, Deutschen, Juden und anderen ernsthaft verletzt worden wären. Dank der gesunden und loyalen Konkurrenz nivellierte sich die rumänische Gesellschaft nach oben.

Dieses hypothetische Drehbuch ist kein steriles Gedankenexperiment.

Die Finnen und die Rumänen haben ihre Nationalstaate in derselben Zeit und unter sehr ähnlichen Bedingungen gegründet. Finnland war Jahrhunderte lang ein Teil Schwedens. Die finnische Gemeinschaft hatte im Augenblick der Staatsgründung eine sehr enge intellektuelle Schicht, für alle Fälle eine viel engere als die Schicht der schwedischen Intellektuellen im neuen Finnland. Die Finnen hielten aber die Vorteile dieser Situation vor Augen, und darum versuchten sie nicht die gegebenen gesellschaftlichen Beziehungen zu verändern. Sie schufen die schwedischen Institutionen nicht ab, sie verboten die schwedische Sprache, assimilierten die schwedische Intellektualität, liquidierten die ökonomische, soziale und kulturelle Konkurrenz nicht. Ganz umgekehrt. Sie haben der Konkurrenz auf Grund des allgemeinen Bilingualismus für die möglichst schnelle Modernisierung der finnischen Sprache, zur Schaffung eines mit dem schwedischen und den anderen europäischen Institutionen vergleichbaren Institutionssystems angewendet. Demzufolge nivellierte sich die finnländische Gesellschaft nach oben. Die Konkurrenz hat die Selbstverwirklichungsmöglichkeiten der finnischen Mehrheit überhaupt nicht verringert. Das hat die optimalen Bedingungen für die Selbstentfaltung aller Staatsbürger Finnlands garantiert. Die Schweden wollten loyale Staatsbürger Finnlands werden.

Rumänien hat leider die andere Möglichkeit gewählt. Der Staat versuchte die ungarischen, deutschen und jüdischen Institutionen in rumänische zu verändern. Der hyperzentralisierte sozialistische Staat bietet dazu fast ideale Möglichkeiten. Die Verstaatlichung aller Gebiete des gesellschaftlichen Seins war ein sehr guter Anlass zur partiellen oder integralen Romanisierung. Es begann die Umbildung der „,ungarischen“ und „deutschen“ Städten in rumänische, des ,uralten ungarischen Bodens“ in „uralten rumänischen Boden“. Die vorher so heftig kritisierte ungarische Technik der Enteignung der sächsischen Städte wurde ohne die geringste Veränderung enteignet. Nach dem Zweiten Weltkrieg sind die Deutschen und die Juden schrittweise ausgewandert. Die Ungarn sind geblieben, aber ohne 
ihre Institutionen, und dem starken Assimilationsdruck ausgeliefert, ist das intellektuelle Niveau der ungarischen Intellektualität unter das der rumänischen gesunken, obwohl der letzte selbst viel unter sein vorheriges Niveau gefallen ist.

Die rumänische Gesellschaft nivelliert sich nach unten.

Die antiminoritäre Politik lässt sich mit demokratischen Argumenten keineswegs legitimieren. Die demokratischen Institutionen der lokalen Selbstverwaltung hatten die voluntaristische Umänderung der siebenbürgischen Städte ganz unmöglich gemacht. Die spontane Veränderung der ethnischen Komposition war durchaus unvermeidbar, weil ein Teil der siebenbürgischen Großstädte von rumänischer Mehrheit besiedelten Gebieten umgeben ist. Diese Verwandlungen wären aber auch natürlich gewesen, weil sie keineswegs zum radikalen und antidemokratischen Durcheinander der geerbten interethnischen Beziehungen führten. Die zweite Alternative machte aber die übertriebene Zentralisierung auch zwanghaft erforderlich. Die Elite konnte die - von den Ungarn übernommenen und auch wesentlich vervollkommnen - antidemokratischen Maßnahmen nur mit mehr oder minder fiktionalen geschichtlichen Theorien rechtfertigen.

Wenn sich die Schöpfer Großrumäniens für die sogenannte „historische Legitimität" entschieden haben, sahen sie sich gezwungen ihre Politik auf die nationale Kirche und Armee, auf autoritäre politische Institutionen, und auf eine „,nationale Metaphysik”, kurz auf die eigentümlichen Requisiten der rechtsradikalen Politik zu fundieren. Die Finnen haben im Gegenteil ein System der bürgerlichen Werte geschaffen, das ihr Land in eine hoch entwickelte westliche Demokratie (noch dazu eine der fortgeschrittenen!) verwandelte.

Die rumänische Intellektualität hat aber nicht ganz frei entschieden. Es ist wahr, dass theoretisch sowohl die rumänischen Intellektuellen, als auch die Finnen zwei entgegengesetzte Alternativen vor sich hatten. Aber haben sie auch zwei entgegengesetzte Praxismodalitäten hinter sich?

Einerseits sah der größte Teil der siebenbürgischen Rumänen die Lösung des nationalen Problems durch die territorialen Autonomien oder durch die Föderalisierung der Österreich-Ungarischen Monarchie. Das beste Beispiel dafür ist das Buch von Aurel C. Popovici: Die Vereinigten Staaten von Groß-Österreich, das 1906 in Berlin erschien, in dem der Verfasser das Projekt einer modernen Staatsföderation erarbeitet. Siebenbürgen wurde in diese Föderation als ein selbstständiger rumänischer Mitgliedstaat eingegliedert. Aber träte auch Szeklerland, das von einer ungarischen Mehrheit besiedelte siebenbürgische Gebiet in der Mitte des heutigen Rumäniens als selbstständiger Mitgliedstaat (der zwölfte) in die Staatsgemeinschaft ein, so bekämen auch die im rumänischen Mitgliedstaat lebenden deutschen weitreichende Autonomien.

Andererseits hatten die Rumänen aus dem Alten Königtum, dem sogenannten Vechiul Regat, eine Reihe von sehr erfolgreichen Assimilierungsversuchen hinter sich. Ein einziges Bespiel: Dobrogea war am Ende des 19. Jahrhunderts eine echte 
multiethnische Region, in der 1908 noch viele Türken und Tataren (über 38 000), Russen (über 37 000), Bulgaren (über 37 000), Lipovenen (eine andere slawische Population, über fast 13 000), Griechen (über 9 000) Deutsche (über 7 000), Juden (über 4 000) und Gagausen, Armenier, Zigeuner, Italiener usw. lebten. Die Rumänen mit ihren 101000 Einwohnern, die die relative Mehrheit bildeten, waren damals noch in absoluter Minderheit. (Şăineanu 1908: 869) Zwischen dem Unabhängigkeitskrieg (1877) und dem Zweiten Weltkrieg wurde die kulturelle Vielfalt der Region völlig zerstört. Heute sind schon 95 Prozent der Einwohner Rumänen.

Die Entscheidung zwischen zwei verschiedenen Alternativen war aber nicht nur Verantwortung der rumänischen Intellektuellen und Politiker. Die entscheidende Rolle hat auch in dieser Hinsicht der Westen gespielt, der Westen, der nach dem Weltkrieg kategorisch, obwohl nicht ausgesprochen, aber in „reinen“, das heißt frankreichähnlichen Nationalstaaten dachte.

Unter solchen Umständen ist es keine Überraschung, dass Rumänien die zweite Alternative gewählt habe.

Der Westen kritisiert heute die wirklich paralysierende Korruption in Rumänien, aber er will sich dessen nicht bewusst machen, dass diese Korruption - neben der türkisch-griechischen politischen Erbe $^{2}$ - eine der härteren Folgen der legitimierten nationalen Ungleichheit in der rumänischen Gesellschaft sei. Diese Ungleichheit und der oft unverhüllte Assimilationsdruck wurden vom Westen tacit akzeptiert.

Auf den Seiten der besten kulturellen Wochenzeitung Rumäniens, Dilemma, fand vor einigen Jahren eine heftige Debatte über den Nationalismus der rumänischen Intellektuellen statt. Ein berühmter ungarischer Publizist und Philosoph, Tamás Gáspár Miklós siebenbürgischer Herkunft, der in den siebziger Jahren in Bukarest klassische Philologie studierte, und sich Freunde unter den berühmtesten und einflussreichsten rumänischen Intellektuellen machte, stellte in einem Offenem Brief die Frage, warum waren und sind auch heute noch die rumänischen Intellektuellen praktisch ohne bedeutende Ausnahme von den rechtsradikalen Ideen fasziniert? Die wahrscheinlichste Antwort war, dass sie von ihren Umständen, in erster Reihe von ihren irrtümlichen originellen Entscheidungen auf diese Ideologie, auf diese Legitimationsmitteln kategorisch angewiesen sind. Diese Fehlentscheidungen waren auch in spiritueller Hinsicht paralysierend. Nach dem Zweiten Weltkrieg konnten geistlich nur jene rumänischen Intellektuellen sich völlig entfalten, die das Land verließen. Ionescu (oder wie ihn die Franzosen nennen: Ionesco), Cioran, Eliade, Brâncuşi (oder wie ihn die Franzosen nennen: Brancusi) konnten nur im Ausland weltberühmt werden. Die ihnen ähnlichen Talente als Noica, Liiceanu und andere mochten nicht das entsprechende Lebenswerk schaffen. Und keineswegs der rumänischen Sprache wegen! Sie alle opferten irgendwie die geistige Selbständigkeit auf dem Altar der nationalen Begeisterung.

2 Die Türken übten in den eroberten rumänischen Prinzipaten die politische und administrative Macht durch griechische Repräsentanten aus, die sogenannten Fanarioten. 
Sie sind natürlich keine Ausnahme gewesen, das gleiche ist (mindestens bis in den letzten Jahren der Diktaturen) fast allen Intellektuellen aller mitteleuropäischen Kleinstaaten passiert.

Dieser falsche Legitimationsdruck begann nur nach der Revolution von 1989 sich aufzuheben. Die neue Demokratie, die von Anfang an die rumänische Macht zum Adoptieren einer ganzen Reihe von echten demokratischen Maßnahmen gezwungen hat, stieß in den ersten Jahren nach der Revolution auf erbitterten Widerstand. Anfang 1990 fanden in Tg. Mureş auch blutige Auseinandersetzungen statt. Die internationale Öffentlichkeit und die Politiker der Großmächte haben es unmissverständlich gemacht, dass sie keine einseitige Lösung akzeptieren wollen und können, käme das von jeglicher Seite.

Es wurde klar, es gibt einen einzigen Weg, den der politischen Verhandlungen, der wechselseitigen Kompromissen und der Zusammenarbeit.

Am Anfang der 90-er Jahre war es unvorstellbar, dass Ungarn und Rumänen in der politischen Wettkampf zusammenarbeiten. Damals gab es eine Metapher, der gemäß wir Ungarn in einer belagerten Burg leben und wer mit dem Belagerer verhandelt, hatte seine Mitbürger verraten. Heute ist das, Gott sei Dank, Vergangenheit. Wir haben noch sehr viele ungelöste Angelegenheiten, aber heute glauben wir, Rumänen, Ungarn und die anderen Minderheiten, dass diese in der nahen Zukunft schon lösbar sind.

Die Identität der rumänischen Staatsbürger, der Rumänen und der anderen nationalen Gemeinschaften, verändert sich augenscheinlich. In der neuen äußeren und inneren Situation ist die wichtigste Eigenschaft der rumänischen Politik der Handelszwang. ${ }^{3}$ Die Ungarn haben Rumänien endlich als ihren Vaterland akzeptiert, und Rumänien versucht sie als wirklich gleichberechtigte Staatsbürger zu integrieren. Das ist nicht problemlos. Die Nationalisten von beiden Seiten versuchen diese Bestrebungen zu scheitern. In hiesiger Lage sind sie aber ab ovo zum Scheitern verurteilt.

Die rumänische Identität beginnt auch seine schönsten Eigenschaften zu offenbaren.

Ich muss zum Schluss den sogenannten „großen Verräter“, den „letzten Fanarioten“, Ion Luca Caragiale zitieren: „Warum erschrecken wir uns, warum grübeln wir vergeblich. Bei uns gibt es nicht mehrere und nicht mindere Verderbtheit als an anderen Teilen der Welt. Und das ist auch natürlich. Die menschlichen Tugenden und die Fehler sind überall dieselben. Sprache, Tracht, Gewohnheit, intellektuelle und konfessionelle Veranlagungen, als auch alle andere Ergebnisse des Ortes wo sie gelebt haben, die Angelegenheiten, die sie in der Vergangenheit erlebt haben, möchten zeigen, dass obwohl die Menschen der verschiedenen Länder sehr verschieden sind, sind sie aber im Grunde überall dieselben. Es gibt kein anderes zoologisches Wesen auf der Welt, der einheitlicher wäre als der König der Schöpfung. Zwischen 
einem polynesischen Menschenfresser und einem hochrafinierten Europäer gibt es einen einzigen klaren Unterschied, der Weise wie sie seine Nahrung fertigt. Keine Rasse ist besser oder böser, intelligenter oder dummer, einer ist solcherart, anderer derartig, letztes Ende aber sind alle gleich. Nenne ihn Mensch und gib ihm Ruhe!

Also grübeln wir und erschrecken wir uns nicht, und denken wir nicht, dass die rumänische Welt verderbter als andere sei. Nein, kategorisch nicht, diese Nation ist nicht verderbt, sie ist noch ungemacht, sie hat bis jetzt nicht genug herangereift. Sie ist von den Elenden unter denen Stöße sie Jahrhunderte lang gestöhnt hat, noch nicht gereinigt, sie glaubt noch nicht in der Wahrheit, sie ist noch nicht imstande seine Anführer zu gebären, sie kennt noch nicht, wen sie anhören müsste, weil sie einstweilen zu niemandem Vertrauen haben kann. Von der Milch verbrannt, wird sie auch den Käse anblasen... (Fript cu lapte, suflă şi-n brînză)“. ${ }^{4}$

Es scheint einem, dass die Europäische Union die wichtigsten Bedingungen anbieten möchte, die zum Heranreifen nötig sind.

\section{Literaturverzeichnis}

Anderson, Benedict 1991. Imagined Communities: Reflections on the Origin and Spread of Nationalism. London-New York, Verso.

Dubar, Claude 2000. La crise des identités: L'interpretation d'une mutation. Paris, Presses Universitaires de France.

Caragiale, Ion Luca 1994. Despre lume, artă şi neamul românesc (ed. Dan C. Mihăilescu). Bucureşti, Editura Humanitas.

Connor, W. 1972. Nation-Building or Nation Destroying? World Politics, vol. 24. 319-355.

Held, David 1995. Democracy and the Global Order - From the Modern State to Cosmopolitan Governance. London, Polity Press.

Kürti, László 2001. The Remote Borderland, Transylvania in the Hungarian Imagination. State University of New York Press.

Kymlicka, Will-Straehle, Christine 1999. Cosmopolitanism, Nation-states and Minority Nationalism: A critical Rewiew of Recent Literature. European Journal of Philosophy, Vol. \&. Number 1, 65-88.

Man, Gloria 1996. The Ortodox Fault Line. Capricorn.

Miskolczy, Ambrus 1994. Lélek és titok (Geist und Geheimnis). Budapest, Kortárs Kiadó.

Patapievici, Horia-Radu 2004. Discernământul modernizării, 7 conferințe despre situația de fapt. Bucureşti, Humanitas.

Şăineanu, Lazăr 1908. Dicționar universal al limbii române. (ed. I. Samitca şi D. Baraş) Craiova.

4 Caragiale 1994, 133-134. 